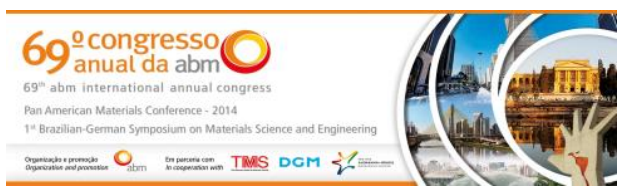

Tema: Diagramas de fases e transformações de fases

\title{
TEXTURA DE TRANSFORMAÇÃO DA MARTENSITA INDUZIDA POR DEFORMAÇÃO EM AÇOS INOXIDÁVEIS AUSTENÍTICOS*
}

\begin{abstract}
Resumo
Aços inoxidáveis austeníticos com baixa energia de falha de empilhamento estão sujeitos à transformação martensítica quando deformados a frio. Diversos autores relatam que esta transformação segue as relações de orientação de Kurdjumov-Sachs ou NishiamaWassermann. Bhadeshia e outros autores vêm sistematicamente contrapondo estas afirmativas mostrando que esta transformação segue a teoria clássica de formação da martensita. A transformação martensítica requer a existência de um plano invariante e um plano de hábito e não apenas uma mudança de orientação como mostrado em muitos trabalhos. Neste trabalho foram utilizados resultados obtidos por ebsd de amostras de aço inoxidável AISI301 deformadas por tração para análise da cristalografia da transformação martensita no nível de microtextura bem para análise de macrotextura, foram usados dados publicados na literatura para um aço AISI 304 cujas medidas de textura em ambas as fases e as frações volumétricas das orientações preferenciais foram realizadas com precisão usando micro-difração de raios-X em um sincrotron. Em ambos os casos os resultados experimentais de textura da fase transformada foram comparados com resultados calculados usando a teoria clássica da transformação martensítica (PMTC) e usando as relações de Kurdjumaov-Sachs (KS) e Nishiyama-Wassermann (NW). Admitiu-se que a seleção de variantes seguiu o modelo de Patel-Cohen.
\end{abstract}

Hamilton Ferreira Gomes de Abreu ${ }^{1}$ Regina Coeli Araujo Vieira ${ }^{2}$ Neuman Fontenele Viana ${ }^{3}$ Miloslav Beres ${ }^{4}$

Palavras-chave: Transformação martensítica; Cristalografia; Relações de orientação.

\section{TRANSFORMATION TEXTURE IN STRAIN-INDUCED MARTENSITE AUSTENITIC STAINLESS STEELS}

\begin{abstract}
Austenitic stainless steels with low stacking fault energy are subject to martensite transformation when cold-deformed. Several authors report that this transformation follows the orientation relationships of Kurdjumov - Sachs or Nishiama - Wassermann. Bhadeshia and other authors have systematically countering those statements showing that this transformation follows the classical theory of the formation of martensite. The martensitic transformation requires the existence of an invariant plane and a plane of habit and not just a change of direction as shown in many studies. In this work, the results obtained by EBSD in an AISI301 stainless steel samples deformed in a tension test were used to analyze microtexture. To analyze macrotexture data for AISI 304 steel texture were accurately measured using micro - X-ray diffraction in a Synchrotron and published in the literature used. In both cases the experimental results of phase transformed texture were compared with results calculated using the classical theory of martensitic transformation (PMTC) and using the relations Kurdjumaov -Sachs (KS) and Nishiyama - Wassermann (NW). It was assumed that the selection of variants followed the model of Patel - Cohen.
\end{abstract}

Keywords: Martensitic transformation; Crystallography; Orientation relationship.

1 Engenheiro Naval, DSc., Professor Associado, Depto. Eng. Metalúrgica e de Materiais, Universidade Federal do Ceará, Fortaleza (UFC), CE, Brasil; hamilton@ufc.br.

2 Graduanda Eng. Metalúrgica, Depto. Eng. Metalúrgica e de Materiais, UFC, Fortaleza, CE, Brasil.

3 Físico, Doutorando Eng. Materiais, Depto. Eng. Metalúrgica e de Materiais, UFC, Fortaleza, CE, Brasil.

4 Engenheiro de Materiais, PhD, Professor Visitante, Depto. Eng. Metalúrgica e de Materiais, UFC, Fortaleza, CE, Brasil.

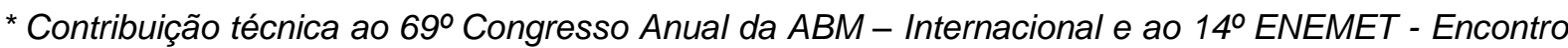
Nacional de Estudantes de Engenharia Metalúrgica, de Materiais e de Minas, 21 a 25 de julho de 2014, São Paulo, SP, Brasil.
} 


\section{INTRODUÇÃO}

Deformação pode induzir a transformação martensítica em aços inoxidáveis . A quantidade de martensita depende de parâmetros de processamento, tais como o estado de tensão do material, a temperatura e a taxa e grau de deformação. A composição química do aço e energia de falha de empilhamento também podem exercer grande influência [1-7]. Quando tensões externas são aplicadas o trabalho realizado contribui para a variação da energia livre, ou aumentando ou diminuindo a temperatura Ms [8] .

Existem duas relações bastante difundidas no estudo da transformação da austenita para a martensita em aços. A relação de Nishiyama-Wasserman (NW) que especifica que $\{110\}_{c c c} /\{111\}_{c f c},<001>$ ccc $/<101>$ cfc. A de Kurdjumov-Sachs (KS) por sua vez estabelece que $\{110\}_{\mathrm{ccc}} /\{111\}_{\mathrm{cfc}},<111>\mathrm{ccc} / /<101>\mathrm{cfc}$. Os resultados destas duas relações diferem de cerca de $5,6^{\circ}$ no plano de interface.

De acordo com Bhadeshia, as relações Kurdjumov-Sachs e / ou NishyamaWassermann não são suficientes para representar a transformação de austenita em martensita. Ao considerar as transformações martensíticas, é necessário não só considerar a relação de orientação, mas também a presença de um plano de hábito e uma deformação. Este conjunto está ligado matematicamente e deve ser considerado como um todo, em análise de textura. A teoria também prevê que as orientações exatas Kurdjumov-Sachs e Nishiyama-Wassermann são impossíveis para transformações em aço porque eles não levam à existência da linha invariante necessário a transformação. A relação de orientação verdadeira é de fato irracional em todos os casos. Embora a diferença entre esta orientação irracional e a obtida com as relações NW e KS possam parecer pequenas (uns poucos graus), é vital porque a ausência de uma linha de invariante entre a fase mãe e o produto faria transformações martensítica impossível [10-14].

Muitos estudos têm sido publicados sobre a deformação martensita induzida com foco na análise de cristal único, ou seja, microtextura. A dificuldade para os estudos macrotextura é que apenas pequenas quantidades de martensita são formadas durante deformação a ser medida através de métodos de difração raios- $X$ ou de nêutrons. Além disso, dados de boa qualidade para as figuras de pólo de construção muitas vezes não estão disponíveis. Fontes de sincrotron moderno, que combinam alta intensidade, tamanho pequeno de feixe e livre escolha do comprimento de onda pode resolver esta dificuldade proporcionando uma boa análise de textura quantitativa e resultando em uma macrotextura precisa de martensita induzida. Um trabalho publicado por Grigull [9] estudou a martensita induzida por deformação em um AISI 304 laminado e recozido expostos a um teste de tração uniaxial. Medições de textura foram realizadas no anel síncrotron Advanced Photon Source no Argonne National Laboratory em energia do feixe de $85 \mathrm{keV}$ e diâmetro de $0,2 \mathrm{~mm}$.

Neste trabalho, utilizou-se uma amostra de AISI-301 deformada por tração para estudo da microtextura. A Macrotextura foi analisada usando resultados publicados na literatura por Grigull [9]. Em ambos os casos, microtextura e macrotextura, comparou-se os resultados calculados usando a teoria fenomenológica de cristalografia da martensita (PMTC), Kurdjumaov-Sachs (KS) e NishiyamaWassermann (NW) com resultados obtidos por ebsd em uma amostra de aço inox no caso da microtextura e para a macrotextura com os resultados obtidos por Grigull no sincrotron.

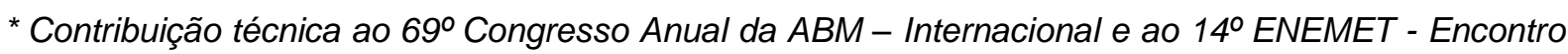
Nacional de Estudantes de Engenharia Metalúrgica, de Materiais e de Minas, 21 a 25 de julho de 2014, São Paulo, SP, Brasil.
} 


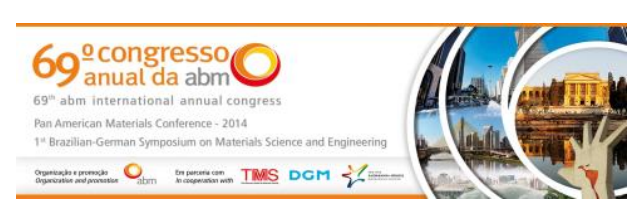

\section{MATERIAIS E MÉTODOS}

Os estudos de EBSD foram realizados em amostra retirada do plano médio da zona deformada de um corpo de prova de tração. Um sistema de EBSD Oxford Cristal 300 acoplado a uma microscópio eletrônico Philips XL-30 foi utilizada e os dados analisados com o auxílio do software HKL-Channel-5.

Para os cálculos com a teoria fenomenológica foram usados os parâmetros de rede da austenita e da martensita $0,3589 \mathrm{~nm}$ e $0.2873 \mathrm{~nm}$ respectivamente, os mesmos utilizados por Kundu (10) para um aço inoxidável AISI 304 [11].

As medidas de textura do trabalho de Grigull foram realizadas em aço inoxidável AISI 304 comercial laminadas e recozidas, deformado em um teste de tração com deformação verdadeira $\varepsilon=0,3$. O valor estimado de tração para esta condição foi entre 700 e $800 \mathrm{Mpa}$.

A textura inicial da austenita foi assumida como a medida por Grigull, composto por três componentes: cobre $(\{211\}<100>)$, Goss $(\{001\}<110>)$ e bronze $(\{011\}<211>)$, com respectivas fracções de volume de 0,70,0,23 e 0,07. Tendo esta informação como ponto de partida, um arquivo de orientação composto por 500 grãos de austenita com textura de acordo com as frações de volume dado foi gerado. Para reproduzir a teoria fenomenológica da transformação martensitica foi utilizado o programa crystal_habit_poly.f, disponível no enderêço ww.msm.cam.ac.uk/phasetrans. Os detalhes sobre a utilização deste software estão disponíveis na referência [15].

\section{RESULTADOS E DISCUSSÃO}

Os resultados obtidos usando a PMTC para plano de hábito, matriz deformação a matriz de transformação estão mostrados a seguir:

Plano de hábito - (-0:183989 0:596344 - 0:781359)y

Matriz deformação:

$$
(\gamma \mathrm{P} \gamma)=\left\{\begin{array}{ccc}
0.991342 & 0.028064 & -0.036770 \\
0.028064 & 0.909040 & 0.119180 \\
0.029429 & -0.095386 & 1.124979
\end{array}\right\}
$$

Matriz transformação:

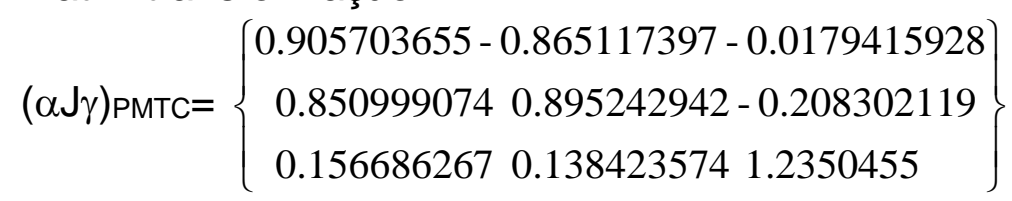

Para as transformações KS e NW as matrizes de transformação usadas foram [16]:

$$
(\alpha J \gamma)_{\mathrm{KS}}=\left\{\begin{array}{ccc}
0.983200014 & 0.166700006 & 0.0749000013 \\
-0.166700006 & 0.649800003 & 0.741599977 \\
0.0749000013 & -0.741599977 & 0.666700006
\end{array}\right\}
$$

\footnotetext{
* Contribuição técnica ao $69^{\circ}$ Congresso Anual da ABM - Internacional e ao 14ํㅡㄹ ENEMET - Encontro Nacional de Estudantes de Engenharia Metalúrgica, de Materiais e de Minas, 21 a 25 de julho de 2014, São Paulo, SP, Brasil.
} 


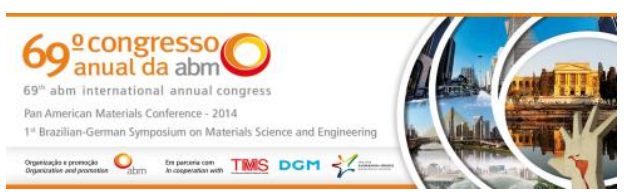

$$
(\alpha J \gamma)_{\mathrm{NW}}=\left\{\begin{array}{lll}
0.985599995 & 0.11959999 & 0.119599998 \\
-0.16910000 & 0.69690001 & 0.69690001 \\
0 . & -0.707099974 & 0.707099974
\end{array}\right\}
$$

A figura a seguir é uma imagem de ebsd para a fase austenita de um aço inoxidável AISI $301 \mathrm{~L}$ com baixa deformação (<10\% na espessura) para permitir a utilização do ebsd. As regiões na cor branca representam a martensita induzida por deformação. A orientação da austenita do grão verde da figura é dada pelos ângulos de Eules $\varphi_{1}=357,1, \phi=36,7$ e $\varphi_{2}=81,6$.

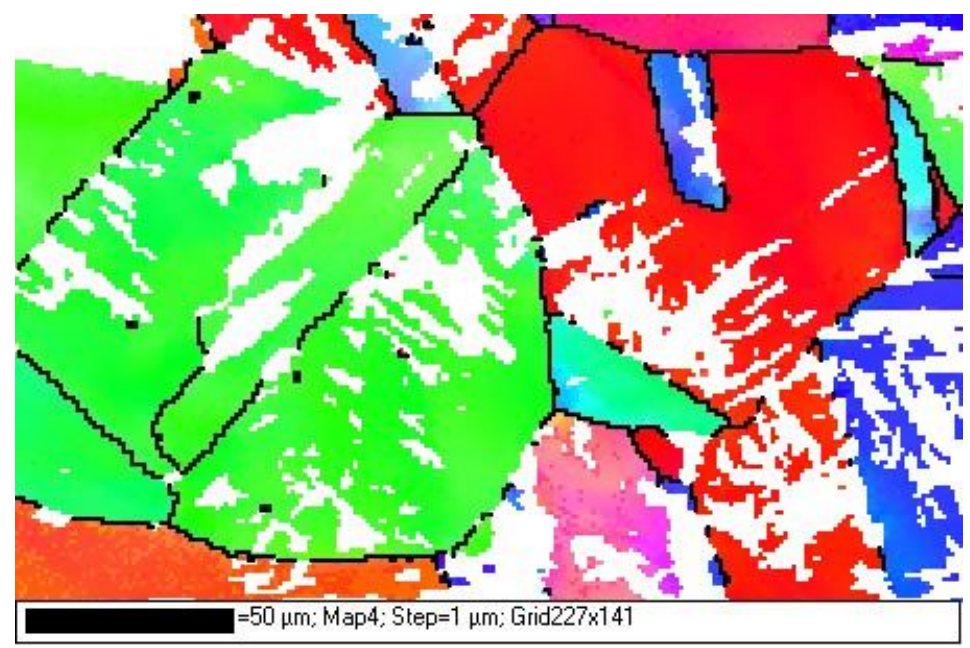

Figura 1- Mapa de orientações obtido por ebsd em amostra deformada por tração.

A figura 2 apresenta a figura de polos $\{100\}$ referente a martensita transformada no interior ao grão verde na parte esquerda da figura. Esta figura deve ser comparada com as três figuras de polos apresentadas na figura 3. A figura 3(a) corresponde a transformação calculada usando a PMTC. A figura 3(b) a relação KS e 3(c) a relação NW. Nos três casos são apresentadas as 24 variantes possíveis para a PMTC e KS e 12 para o caso da NW. Pode-se observar que as figuras de polos calculadas usando a PMTC e KS são bem próximas da figura de polos medida (figura 2). Apenas a título de ilustração, as figuras de polos (100) calculadas pela PMTC (círculos pretos)e as calculadas usando as relações de KS (círculos vermelhos) estão sobrepostas. É possível se verificar como são próximas as diversas variantes.

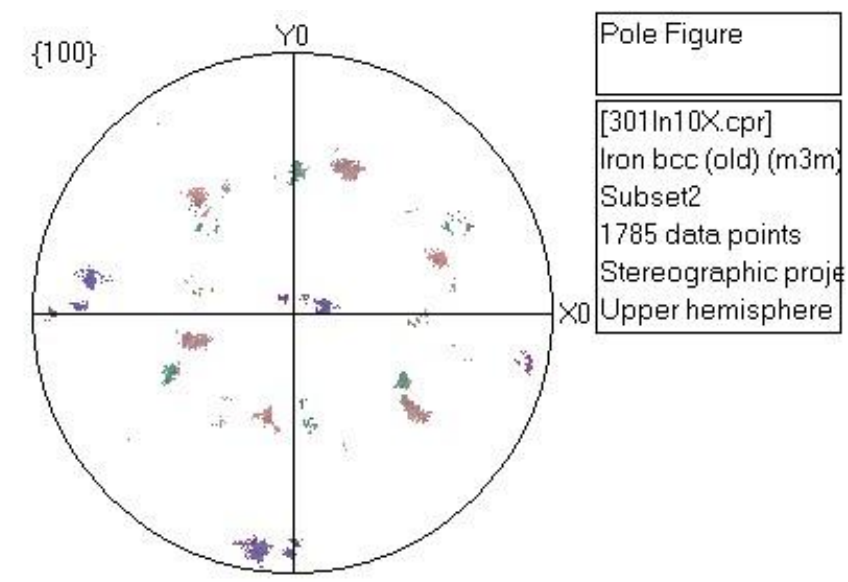

Figura 2- Figura de polos direta para a martensita interior ao grão verde da figura.

\footnotetext{
* Contribuição técnica ao $69^{\circ}$ Congresso Anual da ABM - Internacional e ao 14ํㅡㄹ ENEMET - Encontro Nacional de Estudantes de Engenharia Metalúrgica, de Materiais e de Minas, 21 a 25 de julho de 2014, São Paulo, SP, Brasil.
} 

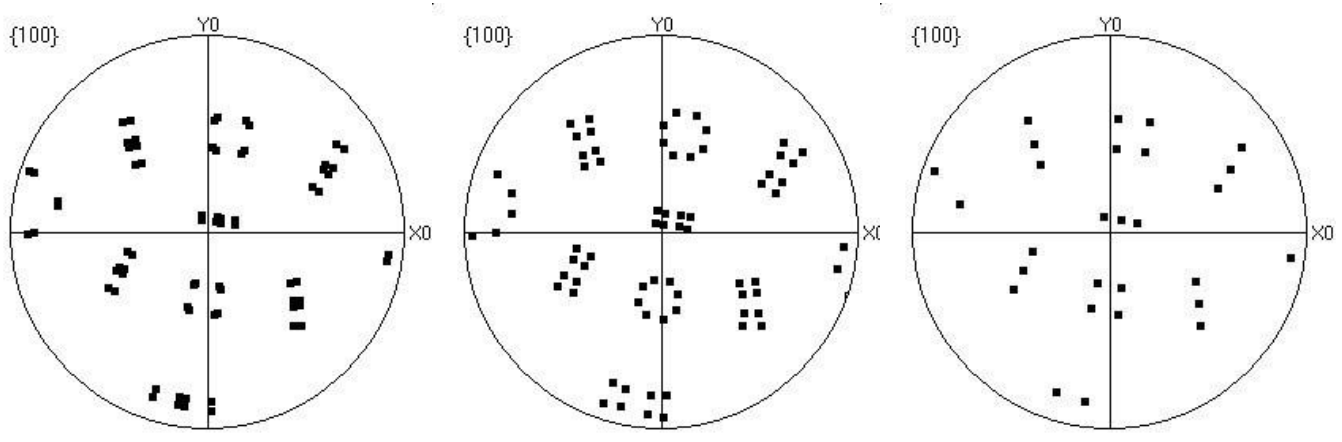

Figura 3 - Figuras de polos calculadas usando (a) PMTC; (b) KS; e. (c) NW.

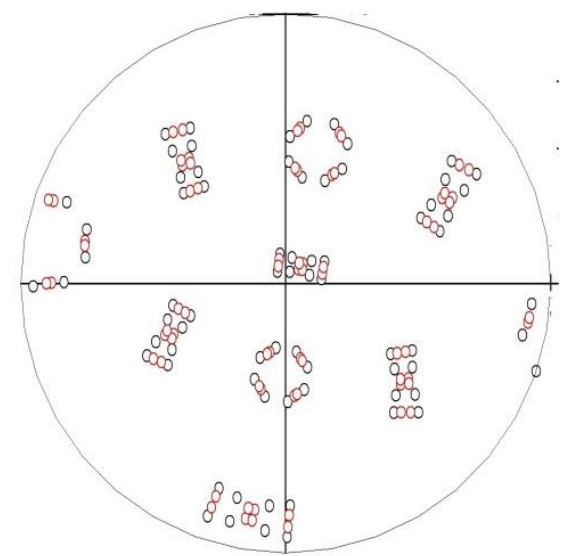

Figure 4 - Figura de polos KS (preta) sobreposta PMTC (vermelha)

A figura 5 mostra a função de distribuição de orientação cristalográfica (fdoc) da austenita gerada a partir das frações volumétricas das orientações medidas por Grugill [9] (70\% orientação cobre, 23\% orientação Goss e 7\% orientação latatão) usando radiação síncroton.

Esta fdoc foi gerada para um conjunto de 500 grãos com orientações cobre, Goss e latão com um desvio de até 20 graus respeitadas as frações volumétricas de cada componente de textura.

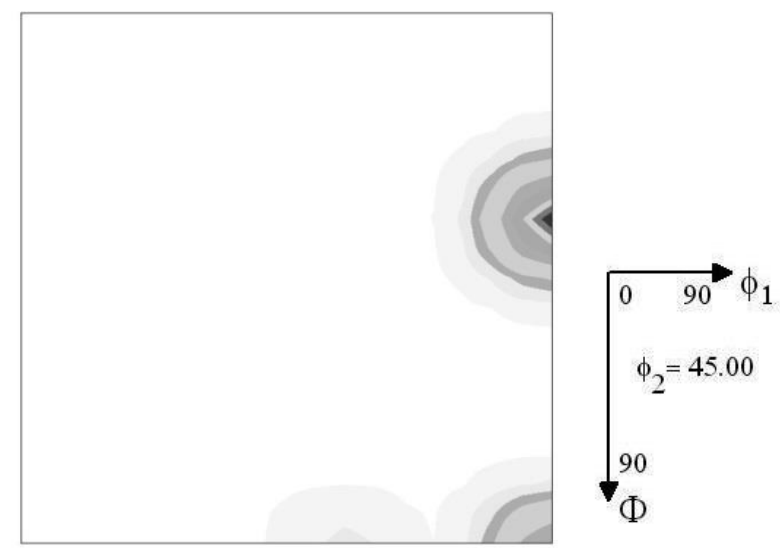

Figura 5 - Seção $\varphi 2=45^{\circ}$ da função de distribuição de orientação cristalográfica para austenita.

A orientação da martensita transformada $(X \mathrm{~J} \alpha)$ foi obtida pelo produto $(\mathrm{XJ} \gamma)(\gamma \mathrm{J} \alpha)$. A seleção de variantes foi feita utilizando o modelo de Patel-Cohen, isto é, apenas as

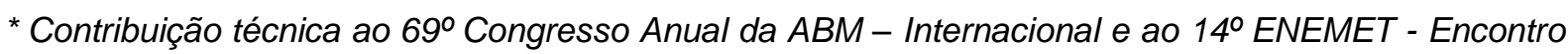
Nacional de Estudantes de Engenharia Metalúrgica, de Materiais e de Minas, 21 a 25 de julho de 2014, São Paulo, SP, Brasil. 


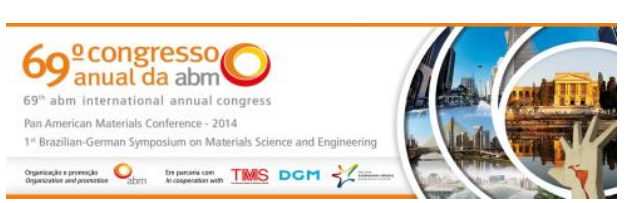

\section{REFERÊNCIAS}

1 Padilha AF, Rios PR. Decomposition of Austenite in Austenitic Stainless Steels. ISIJ International, 2002; 42(4): 325-37.

2 Lecroisey F, Pineau A. Martensitic transformations induced by plastic deformation in the Fe-Ni-Cr-C system. J. Metall Trans., 1972; 3: 387-96.

3 Olson GB, Cohen M. Kinetics of strain-induced martensitic nucleation. Metall Trans A., 1975; 6A:791-5.

4 Olson GB, Cohen M. Stress-assisted isothermal martensitic transformation: Application to TRIP steels. Metall Trans A. 1982;13A: 1907-15.

5 Blanc G, Lacombe P, Baroux B, Beranger G. Stainless Steels - Les Editions de la Physique Les Ulis, Paris, France, 1993: 595-611.

6 Nagy E, Mertinger V, Tranta F, Sólyom J. Deformation induced transformation in stainless steels. Materials Science and Engineering A, 2004; 378A: 308-13.

7 Choi J-Y, Jin W. Strain induced martensite formation and its effect on strain hardening behavior in the cold drawn 304 austenitic stainless steels. Scripta Mater., 1997; 36: 99104.

8 Patel JR, Cohen M. Criterion for the action of applied stress in the martensitic transformation. Acta Materialia, 1953; 1: 531-8.

9 Grigull, S., Tensile deformation induced texture transformation in austenitic stainless steel. Textures and Microstructures, 2003; 35(3/4): 153-162.

10 Kundu S, Bhadeshia HKDK. Crystallographic Texture and Intervening Transformations. Scripta Materialia, 2007; 57: 869-872.

11 Kundu S, Bhadeshia HKDK. Transformation texture in deformed stainless steel. Scripta Materialia, 2006; 55: 779-781.

12 Bowles JS, MacKenzie JK. The crystallography of martensitic transformation I. Acta Metall., 1954; 2: 129-137.

13 MacKenzie JK, Bowles JS. The crystallography of martensitic transformation I I. Acta Metall., 1954; 2: 138-147.

14 Bhadeshia HKDH. Geometry of Crystals. $2^{\text {nd }}$ ed., Institute of Materials, 2001.

15 Kundu S. Transformation Strain and Crystallographic Texture in Steels PhD thesis, University of Cambridge, Department of Materials Science and Metallurgy, Cambridge, UK, 2007.

16 Nozle G. Characterization of the FCC/BCC orientation relationship by EBSD using pole figures and variants Z, Metallkd 95, 2004; 9: 744-755.

\footnotetext{
* Contribuição técnica ao $69^{\circ}$ Congresso Anual da ABM - Internacional e ao 14ํㅡㄹ ENEMET - Encontro Nacional de Estudantes de Engenharia Metalúrgica, de Materiais e de Minas, 21 a 25 de julho de 2014, São Paulo, SP, Brasil.
} 